\title{
A Review of 1-Dimensional and 2-Dimensional Leaky Wave Antenna and Recent Advancements in LWAS
}

\author{
Aparna Vashishtha \\ B.Tech, Dept. of ECE \\ DIT University, Dehradun
}

\author{
Akriti Yadav \\ B.Tech, Dept. of ECE \\ DIT University, Dehradun
}

\author{
Dhruv Chaudhary \\ A P, Dept. of ECE \\ DIT University, Dehradun
}

\begin{abstract}
This paper gives a basic review and a summary of old and recent advancements on Leaky Wave Antenna theory and design. Leaky waves have been among the most active areas of research in microwave engineering over the second half of the $20^{\text {th }}$ century. A leaky wave is treated mathematically as a complex plane wave and the resulting radiation pattern is derived in terms of the complex propagation constant. In particular, the compatibility with the printed circuit board technology, their low profile, easiness of fabrication and integration with the other planar components are the strongest features of Leaky Wave Antennas. A LWA uses a guiding structure that supports wave propagation along the length of the structure, with the wave radiating or "leaking" continuously along the structure. Such antennas is classified into two categories, namely one-dimensional and twodimensional variants. They radiate primarily at the end fire direction and broadside direction to get the maximum scan angle of the radiation beam and these antennas may be uniform, quasi-uniform, periodic. These antennas have many applications at the millimetre wavelengths, frequency scanning. In this paper, a summary of some recent advances for these types of structures is given. Recent advances include structures that can scan end fire, structures that can scan broad side, and structures that are conformal to surfaces.
\end{abstract}

\section{Keywords}

Travelling wave antenna; leaky wave antenna; leaky wave; rectangular waveguide; end fire; broad side; frequency scanning

\section{INTRODUCTION}

Leaky wave antenna (LWA) belongs to the travelling wave antenna class. Travelling wave antennas are characterized by a simple feed launching one or more travelling wave modes in a guiding structure. The modes launched in the guiding structure can be classified as "slow" or "fast", if their phase velocity is respectively lower or higher than the free space velocity. Surface waves are examples of slow waves, whereas leaky waves are examples of fast waves. The modes travelling in the guiding structure control the field distribution on the radiating aperture of the antenna and then the radiation mechanism associated to the antenna. A leaky wave arises from a guiding structure with some or other continuous or periodic discontinuity that facilitates energy leakage into the surrounding area. . The illuminated aperture extends over several wavelengths; the longer the aperture, the narrower is the radiation beam. To have radiation, the mode of propagation should be fast mode with phase constant less than free space propagation constant and at the same time it leaks energy in reason of the attenuation constant. The beamwidth becomes narrower as attenuation constant is reduced. In 1940s, LWA consisting of a slotted rectangular waveguide was introduced [4]. The field has been in steady development since then, with particular developments in more recent years being directed toward planar LWAs, which have the advantage of being low profile and easy of manufacture. The purpose of this paper is to review the LWAs classification and the latest developments in the field of LWAs. Readers are referred to [5]-[9] for more background information and history.

In this paper, we review the mathematical treatment of the leaky waves as complex plane waves and derive the radiation pattern in terms of the complex propagation constant in next section. Several leaky wave antenna structures have evolved and analyzed in the last decade. Broadly speaking, leaky wave antennas are categorized as one dimensional and two dimensional. In the 1-D, means that the wave is guided in one direction along the guiding structure [8]. A typical example is a micro strip line that is periodically modulated in order to turn the non radiating micro strip mode into a radiating leaky mode. This type of LWA radiates in a fashion similar to that of a linear array, creating either a fan beam at broadside or a conical beam that radiates at a scan angle $\theta_{0}$ with respect to the axis of the guiding structure. The structure is typically fed at one end so that the wave propagates down the axis of the structure [as shown in Fig. 1(a)], although a centre feed that creates a bidirectional excitation [as shown in Fig. 1(b)] is also possible and is sometimes very useful, especially for creating broadside beams. This particular configuration has been treated theoretically and experimentally [10] and will be reviewed here in section 3. The two dimensional (2-D) leaky wave structures depend on radially travelling waves and they produce pencil beam in the broadside direction and conical beams otherwise. Examples of such antennas include a stack of dielectric layers of contrasting alternate high/low permittivities that are discussed in section 4 .

\section{CHARACTERISTIC OF LEAKY WAVES}

PROPERTIES

As stated earlier a leaky wave occurs from a closed waveguide with some means of continuous or periodic power leakage into the exterior region. An example of leaky waveguide is a rectangular waveguide with a slotted side wall as depicted in Fig. 2.The waveguide is perturbed with periodic slots in the structure at a certain position. The energy leakage mechanism results in the waveguide having a complex propagation wave number. 

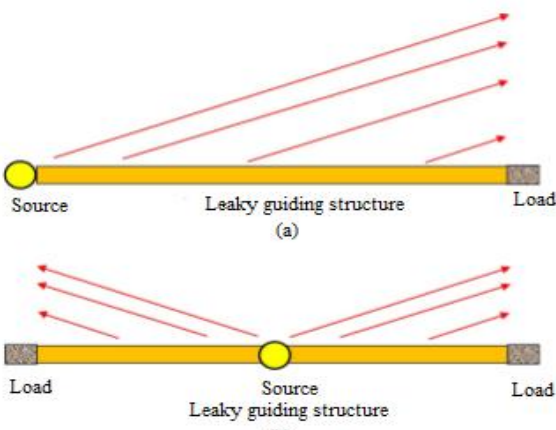

(b)

\section{Fig 1 Illustration of different modes of operation for an} LWA.(a)unidirectional case.(b)bidirectional case

The phase constant is given by $\beta$ and the attenuation constant is indicated by $\alpha$. The attenuation constant varies in size depending on the leakage per unit length along the waveguide; a larger value for $\alpha$ means that a larger amount of energy leaks from the structure [9]. As seen from Fig. 2, the complex propagation parameters are dependent on the geometry of the leaky structure. Control over the beam shape and side lobe levels may be obtained by using an appropriate aperture taper. This is accomplished in practice by slowly varying $\alpha$ along the length of the guide in a specific fashion, and simultaneously holding the phase constant at a fixed value.

The entire waveguide in Fig.2 is regarded as the effective aperture of the antenna, unless the energy leakage is so severe that the signal power fades away completely before reaching the end of the slotted section. A large value for $\alpha$ (which indicates a large leakage rate) in turn leads to a smaller effective aperture, and from antenna theory it is known that the smaller aperture would lead to an increase in beamwidth [11]. On the contrary, lower a values result in a much longer effective aperture and thus the antenna would radiate a narrow main beam, if the physical size of the structure permits it

It should be noted that if the aperture is fixed beforehand and the leakage rate $\alpha$ is relatively small, the antenna pattern (particularly the beamwidth) is primarily influenced by the aperture itself, rather than the leakage rate. However, the radiation efficiency in such a case is strongly affected by the leakage rate. A reasonable design goal is to have approximately $90 \%$ of the power in the waveguide radiated from the structure when the wave has propagated through the entire structure. In most practical cases, a matched load will be connected at the end of the waveguide in order to absorb the remainder of the signal power.

Rectangular waveguides support either transverse electric (TE) or transverse magnetic (TM) modes of wave propagation, and for both cases the phase constant of the wave is frequency dependent [12]. As the frequency (phase constant $\beta$ ) changes, so does the direction in which the principal beam is pointing. Therefore, the antenna beam can be scanned by altering the excitation frequency.

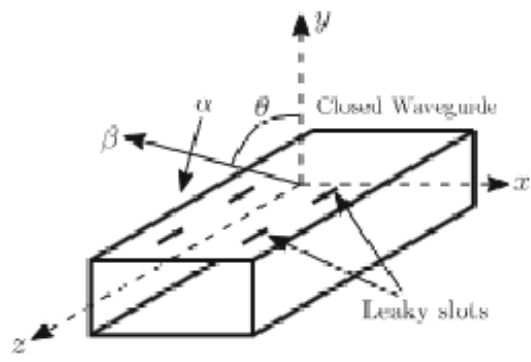

Fig 2- Slotted rectangular waveguide leaky wave antenna

\section{EXTENSION INTO MILLIMETRE WAVELENGTHS}

Leaky-wave antennas have been extensively studied in the last three to four decades, and their main attraction is high directivity, wide bandwidths, and their ability to scan with frequency. Earlier, leaky-wave antennas were based almost exclusively on closed waveguides and the leakage was introduced by cutting holes or slots into the guiding structure. Waveguide losses become exceedingly high at millimetre- wavelengths. As a result, millimetre waveguides are typically open structures, in an attempt to lower the attenuation constant that results from dielectric or metallic losses [13]. Simple open waveguides support the propagation of slow waves, which do not radiate outward from the guide, and the periodic structure is chosen such that only the $n=-1$ space harmonic is able to radiate power. On the other hand, some millimetre waveguides can be closed structures, such as metal guides.

With a basic theoretical background on leaky wave modes established, we will now move on to covering influential designs and concepts that have covered in recent years.

\section{OVERVIEW OF LEAKY WAVE ANTENNAS}

\subsection{Classification of LWAs}

As stated above, a LWA is based on a guiding structure that propagates a wave in one direction, either uni directionally (a source is placed at one end of the structure) or bi directionally (a source is placed in the middle of the structure).Depending upon the geometry, principle of operation and the nature of structural perturbations, leaky wave antennas can be divided into several categories [14]. As mentioned in introduction, the basic distinction is between one-dimensional and two-dimensional leaky wave antennas. Furthermore, these can be divided as periodic, uniform or quasi-uniform structures. These configurations will discussed separately in the succeeding sections.

\subsubsection{One-Dimensional Uniform Leaky Wave Antenna Theory}

A one-dimensional structure is one that supports a wave travelling in a single, fixed direction. In this case, the structure supports a wave that is fast with respect to free space, so that the complex wave number of the leaky mode $k_{z}=\beta-\mathrm{j} \alpha$ has a phase constant in the range $0<\beta<\mathrm{k} 0$. One such structure is a rectangular waveguide with a long uniform slit in one of its side walls shown in fig 3 . The geometry of this guide can be considered uniform along the 
length (taken here as the z-direction). The radiation produced by this structure is limited to the $\mathrm{z}>0$ region, and the resulting beam is in the shape of a cone along the z-axis. As the angle approaches 90 degree or broad side direction, antenna pattern takes on a narrow donut type shape.

It is difficult to achieve a broadside beam if the antenna is fed from only one end, so by feeding the waveguide with a frequency that is marginally higher than the cut off, it may be possible to achieve a broad side beam. This may be accomplished by feeding it from both ends, or by placing the source in the middle of the guiding structure.

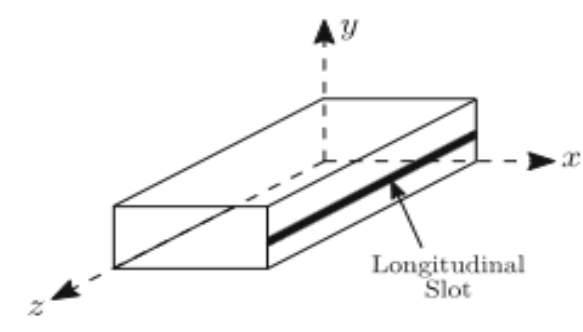

Fig 3 Rectangular waveguide with a longitudinal slot on its side wall

\subsubsection{One-Dimensional Periodic Leaky Wave Antenna Theory}

A one-dimensional periodic antenna comprises a uniform structure that supports a wave that is slow with respect to free space, so that $\beta>k_{0}$ and is modified periodically in the z-direction. In a periodic antenna, the fundamental non radiating mode is made to radiate by introducing the periodicity along the length of the structure in order to produce the radiation. A primary advantage of periodic leaky wave antennas is that the direction in which the beam points could be either forwards or backwards, depending upon the phase constant used in the excitation.

The guide dimensions are chosen such that the dominant mode is the only mode above the cut off frequency, and since $\beta>k_{0}$ for this particular mode, it is purely bound. After the dimensions have been set, the metal strips in Fig 4 are added periodically, and the field on the structure is then characterized by an infinite number of space harmonics having wave numbers [15].

$$
k_{z, n}=k_{z, 0}+2 \pi n / p
$$

Where $\mathrm{p}$ is the period in the $\mathrm{z}$-direction. As the frequency increases, space harmonic reaches a critical point where the $\mathrm{n}=-1$ becomes fast, a beam originates from the backward end fire. Further increasing the frequency will result in the beam scanning from backward end fire, through broad side, up to forward end fire.

A periodic leaky wave antenna therefore radiates as a result of the $n=-1$ space harmonic, which is the fundamental difference between uniform and periodic structures.

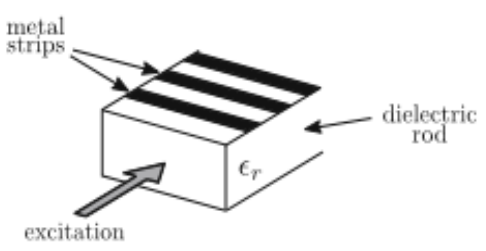

\section{Fig 4 Dielectric rod, periodically loaded with metallic strips}

\subsubsection{Modes of Operation and Basic Principles}

There are four possible operational configurations for 1-D LWAs, since the structures can be uniform/quasi-uniform or periodic, and they can be either unidirectional leaky wave or bidirectional leaky wave. Different types of beams can be obtained depending on the case. Each case is summarised below.

1) Uniform/Quasi-uniform Unidirectional: The structure is fed at one end $(\mathrm{z}=0)$ and typically an absorber or matched load is placed at the other end $(\mathrm{z}=\mathrm{L})$ to absorb residual power, as shown in Fig 1. Along the structure the radiating field or current has the form

$$
\Psi(\mathrm{z})=\mathrm{A} e^{-j k_{z}} Z
$$

Where $k_{z}=\beta-j \alpha$. The radiation efficiency due to the load absorption is $e_{r}=1-\exp (-2 \alpha \mathrm{L})$. A typical choice for the efficiency is around $e_{r}=0.9$ (i.e.90\%), which maximizes the overall gain for a given antenna length L [16].

The structure radiates a conical beam at an angle $\theta_{0}$ from the z-axis, where $\beta=k_{0} \cos \theta_{0}$. The cone of radiation is in the forward direction $\left(0^{0}<\theta_{0}<90^{\circ}\right)$ for $\beta>0$ and in the backward direction $\left(90^{\circ}<\theta_{0}<180^{\circ}\right)$ for $\beta<0$. In this case, a fan beam is produced at broadside. The antenna may be able to radiate at end fire $\left(\theta_{0}=0^{0}\right)$ or backward end fire $\left(\theta_{0}=180^{\circ}\right)$, but this depends on the element pattern of the radiating source. If the element pattern has a null at end fire or backward end fire, then the LWA cannot radiate in these directions. If the structure can radiate at end fire or backward end fire, the beam will change from a conical beam to a pencil beam as forward/backward end fire is approached.

2) Uniform/Quasi-Uniform Bidirectional: The structure is fed in the middle $(\mathrm{z}=0)$ and typically an absorber or matched load is placed at the other two ends $(\mathrm{z}= \pm \mathrm{L} / 2)$ to absorb residual power, as shown in Fig. 1(b). The feed is chosen to excite the radiating field or current symmetrically. Along the structure the radiating field or current then has the form

$$
\Psi(\mathrm{z})=\mathrm{A} e^{-j z} k_{z}
$$

The structure radiates a pair of conical beams pointing at $\theta= \pm \theta_{0}$. The two conical beams will merge into a signal fan beam pointing at broad side $\left(\theta_{0}=90^{\circ}\right)$ whenever $\beta<\alpha$. For those structures based on a rectangular or parallel plate waveguide, the maximum power density radiated at broad side by a simple dipole source inside the structure will occur when $\beta=\alpha$, while the narrowest fan beam will occur when $\beta$ $=0.518 \alpha[17]$.

Periodic Unidirectional: In this case, a periodic LWA is fed at one end $(\mathrm{z}=0)$ and a load is placed at the other end $(\mathrm{z}=$ 
$\mathrm{L}$ ). The fundamental mode is a slow wave, but radiation occurs from the $\mathrm{n}=-1$ space harmonic, which may be forward $\left(\beta_{-1}>0\right)$ or backward $\left(\beta_{-1}<0\right)$. Hence, the LWA can be used to create a beam pointing in either the forward direction $\left(0<\theta_{-1}<90^{0}\right)$ or the backward direction $\left(90^{\circ}<\theta_{-1}<180^{\circ}\right)$. The beam angle will increase as the frequency increases, and hence this type of LWA is often used to scan the beam with frequency. In order to scan the beam from backward end fire to forward end fire in single-beam operation, so that only the $n=-1$ space harmonic radiates, the effective permittivity of the guiding structure must be chosen so that $\varepsilon_{r}^{\text {eff }}>9$ [9]. This is equivalent to the condition that $\frac{\beta_{0}}{k_{0}}>3$. A periodic LWA that has only pure series or shunt radiating elements cannot overcome the stop band problem (at the stop band frequency $\beta_{0 p}=2 \pi$, and hence the impedance seen at the elements will always be an open or short circuit, respectively).

3) Periodic Bidirectional: In this case, the periodic LWA is fed in the centre to create a bidirectional leaky wave, just as for the uniform (case 2) above. This structure radiate at the broadside as a LWA by operating slightly away from the broadside frequency, so that $\beta_{-1}>0$ or $\beta_{-1}<0$. A bidirectional beam is created in which the beam from half of the structure is pointed slightly off of broadside, but the combination of the two beams results in a symmetrical broadside beam. In it, the maximum power radiated at broadside occurs when $\left|\beta_{-1}\right|=\alpha$ [18].

\subsubsection{Two-Dimensional Leaky Wave Antenna Theory}

A two-dimensional leaky wave antenna uses a guiding structure which propagates radially from the feed point. This configuration provides an easy method of obtaining a directive beam of broadside, requiring only a simple source. The general form of such a structure is a partially reflecting surface above a ground structure. This is illustrated in Fig. 5.

The excitation source shown in Fig.5 is a simple horizontal dipole placed within the substrate, at a set distance above the ground plane. The antenna pattern, however, depends on the structure and not on the excitation. The substrate/ superstrate structure could also be extended to include multiple layers of the dielectric material, with the advantage of narrowing the beamwidth [8]. Increasing the permittivity of the superstrate layer results in a narrower beamwidth. Another example of a partially reflecting surface is shown in Fig. 6 .

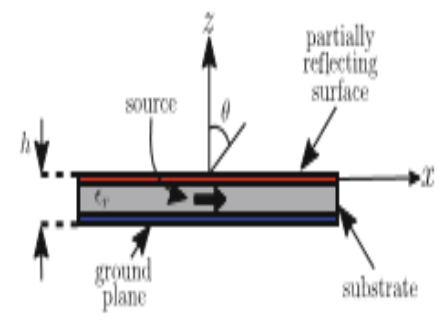

Fig 5 Substrate structure of a 2-D LWA

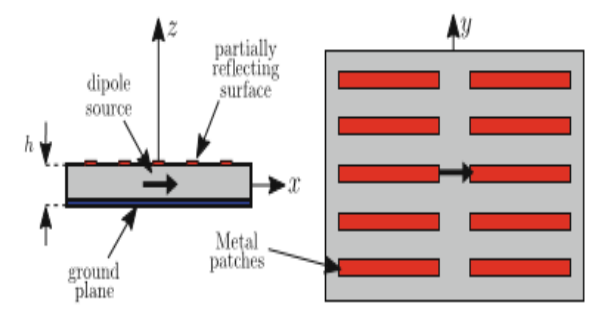

Fig 6 Construction of a partially reflecting surface using metal patches

Larger rectangular micro strip elements will result in a narrower beamwidth. A similar configuration to the one shown in Fig. 6 is to replace the patches with slots. Conversely, smaller slots will result in a narrower beamwidth, and it should be noted that the surface wave feeding the antenna is directed in the y-direction (rather than in the $\mathrm{x}$-direction as with the micro strip array). As the excitation frequency is increased, the pencil beam originating from the antenna will assume a conical shape, effectively losing gain in the broadside direction [19]. Generally, the guiding structure in a two-dimensional leaky-wave antenna is uniform (or at least quasi-uniform) and the excited wave is a fast wave.

\section{RECENT DEVLOPMENTS IN LEAKY WAVE ANTENNAS}

In this section, some recent developments in 1-D LWAs are overviewed. Due to space constraints, only the most relevant properties are summarised, and the reader referred to references for more details.

1) End fire Substrate Integrated Waveguide (SIW) LWA: This is illustrated in Fig. 7 which is one of the recent structure that can produce a pencil beam at end fire. It consists of an SIW waveguide with a periodic array of transverse slots [20]. The SIW waveguide operates in the TE10 mode, and hence the SIW waveguide simply acts as an equivalent rectangular waveguide, made in integrated form. Leakage occurs through the slots, the dimensions of which control the leakage rate (attenuation constant due to leakage). The slots are fairly closely spaced, so the structure is a quasi-uniform LWA, radiating from the slot-perturbed TE10 mode that is a fast wave. The beam radiated would normally be a conical beam, but as discussed in Section 4.1.3,1 the beam becomes a pencil beam at end fire $\left(\theta_{0}=0\right)$ if the element pattern allows for endfire radiation. This is the case here, since the slot fields are equivalent to magnetic currents in the $\mathrm{x}$-direction.

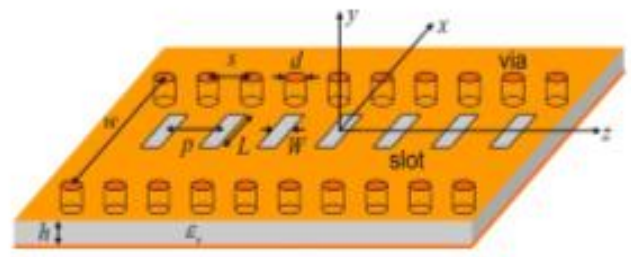

Fig 8 LWA derived from a periodically slotted substrate integrated waveguide 
2) Composite Right/Left Handed (CRLH) LWA: The CRLH LWA led to a breakthrough in LWA design, since it was the first such structure that was able to successfully scan through broadside without beam degradation [21]. As noted in section 4.1.3,3, an open stop band at broadside usually prohibits broadside radiation from a unidirectional periodic LWA. Indeed, the open stop band will always occur when the periodic structure consists of only series or shunt radiating elements, such as a micros trip line loaded periodically with stubs, etc. The CRLH structure is a meta materialinspired design that consists of a periodic arrangement of inductor and capacitor elements within a transmission line unit cell, as shown in Fig. 9. The "right-handed" elements are those that would normally be present in the unit cell of a small length of transmission line. The "left-handed" elements are those that have been artificially added. The resistances account for material loss as well as radiation. The structure is classified as quasi-uniform, since the period is small relative to a wavelength, and hence the structure radiates from the fundamental $n=0$ space harmonic.
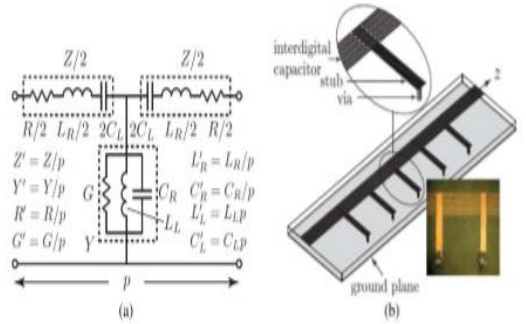

Fig 9 (a) unit cell of the CRLH structure. (b) Practical realization of the CRLH structure.

3) Conformal LWA: In this application, a LWA is put on a curved surface such as a cylinder. The cylinder can be round or of any shape. A three-faced cylinder is shown in Fig. 10 [22]. The goal is to radiate a narrow beam in a fixed direction. Because of the curvature of the cylinder, the LWA should radiate a beam locally at an angle (with respect to the propagation direction of the leaky wave) that changes as the position on the cylinder changes. In this way, the radiation from all points on the LWA is focused in the same direction. Notice that the radiated beam is pointing locally in the backward direction for some points on the cylinder, in the forward direction for other points on the cylinder, and at broadside for one point on the cylinder. This is illustrated in Fig.10. Because the local angle of radiation needs to change with position, and broadside is included, this application requires a LWA that can radiate in both directions and at broadside as well.
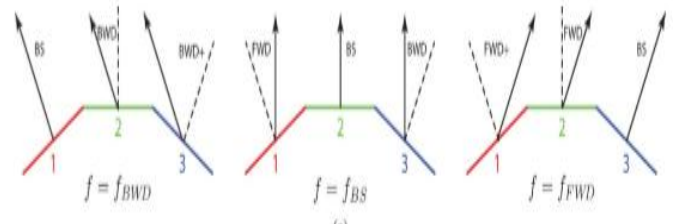

Fig 10 Sketch of a radiating beam for a conformal LWA on a three-sided cylindrical surface

\section{CONCLUSION}

This paper has summarised the state of development of leaky wave antennas, in terms of extending these antennas into the millimetre wavelength region and also the recent developments in the field of LWAs. Although LWAs have been in existence since the 1940s, the field has been significant developments in recent years, with much of the recent work being in the area of planar LWAs, which are low profile and relatively easy to manufacture. Some of the recent developments in the LWA field have been related to the recent developments in the meta materials area, which has provided new inspiration for novel designs. Various designs that overcome the stop band problem at broadside and allow for continuous beam scanning through broad- side have been reviewed. Other developments that have been reviewed include LWAs that can scan to end fire, that can be mounted conformally on a curved surface.

\section{REFERENCES}

[1] Francesco Monticone, student of IEEE, and Andrea Alu, fellow IEEE, "Leaky Wave Theory, techniques, and applications: from microwave to visible frequencies.

[2] Prof. Stefano Maci, Dr. Andrea Neto, in Analysis and design of efficient planar leaky wave antennas.

[3] David R. Jackson, fellow IEEE, Christrope Caloz, fellow IEEE, and Tatsuo Itoh, life fellow IEEE, in " Leaky Wave Antennas" in june 14,2012.

[4] W. W. Hansen, "Radiating electromagnetic waveguide," U.S., Patent 2.402.622, 1940.

[5] A. A. Oliner and D. R. Jackson, "Leaky-wave antennas," in Antenna Engineering Handbook, J. L. Volakis, Ed. New York: McGraw-Hill, 2007.

[6] D. R. Jackson and A. A. Oliner, "Leaky-wave antennas," in Modern Antenna Handbook, C. Balanis, Ed. NewYork:Wiley,2008.

[7] C. Caloz, D. R. Jackson, and T. Itoh, "Leaky-wave antennas," in Frontiers in Antennas: Next Generation Design \& Engineering. New York: McGraw-Hill, Dec. 2011.

[8] A. Sutinjo, M. Okoniewski, and R. H. Johnston, "Radiation from fast and slow travelling waves," IEEE Antennas Propag. Mag., vol. 50, no. 4, pp. 175-181, Aug. 2008.

[9] C. H. Walter, Travelling Wave Antennas. New York: McGraw-Hill,1965.

[10] Mahmoud,S.F., Antar, Y.M, Hammad,H.F., and A.P Freundorfer," Theoretical considerations in the Optimization of Surface waves on a Planar structure",IEEE Trans. on Antennas and Propagation, pp.2057- 2063,August,2004.

[11] A. Oliner, D. Jackson, Leaky-wave antennas, in Antenna Engineering Handbook, 4th edn., ed. by J.Volakis(McGraw-Hill,NewYork, 1969.

[12] C.A. Balanis, Antenna theory: A review. Proc. IEEE80(1),7-23,(1992).

[13] D. M. Pozar, Transmission lines and waveguides, in Microwave Engineering, 4th edn. (John Wiley \& Sons, Inc.,Hoboken,New Jersey, 2012). 
[14] J.L. Gómez-Tornero, A. de la Torre Martínez, D.C. Rebenaque, M. Gugliemi, A. Álvarez-Melcón, Design of tapered leaky-wave antennas in hybrid waveguideplanar technology for millimeter waveband applications. IEEE Trans. Antennas Propag. 53(8), 2563-2577 (2005).

[15] D. Jackson, A. Oliner, Leaky-wave antennas, in Modern Antenna Handbook, ed. by C. Balanis (John Wiley \& Sons, Inc., New York, 2008.

[16] A. Hessel, BGeneral characteristics of travelling-wave antennas,[ in Antenna Theory, Part 2, R. E. Collin and F. J. Zucker, Eds. New York: McGraw-Hill,1969.

[17] E. M .O'Connor,D.R. Jackson, and S.A. Long, "Extension of the Hansen-Woodyard condition for end fire leaky-wave antennas," IEEE Antennas Wireless Propag. Lett., vol. 9, pp. 1202-1204, 2010.

[18] G. Lovat, P. Burghignoli, and D. R. Jackson, "Fundamental properties and optimization of broadside radiation from uniform leaky-wave antennas," IEEE Trans. Antennas Propag., vol. 54, no. 5, pp. 1442-1452, May,2006.
[19] P. Burghignoli, G. Lovat, and D. R. Jackson, “Analysis and optimization of leaky-wave radiation at broadside from a class of 1-D periodic structures," IEEE Trans. Antennas Propag., vol. 54, no. 9, pp. 2593-2603, Sep.2006.

[20] T. Zhao, D.R. Jackson, J.T. Williams, A.A. Oliner, General formulas for 2-d leaky-wave antennas. IEEE Trans. Antennas Propag. 53(11) 3525-3533(2005).

[21] J. Liu, D. R. Jackson, and Y. Long, "Substrate integrated waveguide (SIW) leaky-wave antenna with transverse slots," IEEE Trans. Antennas Propag., vol. 60, no. 1, pp. 20-29, Jan. 2012.

[22] C. Caloz, T. Itoh, and A. Rennings, "CRLH metamaterial leaky-wave and resonant antennas," IEEE Antennas Propag. Mag., vol. 50, no. 5, pp. 2539,Oct.,2008.

[23] M. R. Hashemi and T. Itoh, "Electronically controlled metamaterial-based leaky-wave transmission-line for conformal surface applications," in Proc. Int. Microw. Symp., Boston, MA, Jun. 2009, pp. 69-72. 